\title{
Midlife Intakes of the Isoflavone Genistein and Soy and the Risk of Late-life Cognitive Impairment: The JPHC Saku Mental Health Study
}

\author{
${ }^{1}$ Epidemiology and Prevention Group, Center for Public Health Sciences, National Cancer Center, Tokyo, Japan \\ ${ }^{2}$ Department of Neuropsychiatry, Keio University School of Medicine, Tokyo, Japan \\ ${ }^{3}$ Precision Health, Department of Bioengineering, Graduate School of Engineering, The University of Tokyo, Tokyo, Japan \\ ${ }^{4}$ Department of Clinical Sciences, Lund University, Skåne University Hospital, Malmö, Sweden \\ ${ }^{5}$ School of Health Innovation, Kanagawa University of Human Services, Kanagawa, Japan
}

Thomas Svensson ${ }^{1,2,3,4,5}$, Norie Sawada ${ }^{1}$, Masaru Mimura ${ }^{2}$, Shoko Nozaki ${ }^{2}$, Ryo Shikimoto ${ }^{2}$, and Shoichiro Tsugane ${ }^{1}$

Received April 23, 2021; accepted November 11, 2021; released online December 18, 2021

\begin{abstract}
Background: The number of people with cognitive impairment, including dementia, in the world is steadily increasing. Although the consumption of isoflavones and soy is associated with a reduced risk of cardiovascular disease, it might also be associated with cognitive impairment. The low number of studies investigating the association between soy/isoflavone intake and cognitive function warrant additional research.

Methods: The Japan Public Health Center-based prospective (JPHC) Study is a large population-based cohort. Midlife dietary intake of soy and the isoflavone genistein was assessed on two occasions: in the years 1995 and 2000. In 2014-2015, 1,299 participants from Nagano prefecture completed a mental health screening. Of these, a total of 1,036 participants were included in analyses. Logistic regression was used to determine Odds Ratios (OR) and 95\% Confidence Intervals (CI) for the association between midlife energy-adjusted genistein and soy food intake and cognitive impairment.

Results: There were 392 cases of cognitive impairment (346 cases of MCI and 46 cases of dementia). Compared to the lowest dietary quartile of energy-adjusted genistein intake, the highest quartile was significantly associated with cognitive impairment $(\mathrm{OR}=1.51 ; 95 \% \mathrm{CI}, 1.02-2.24 ; P$ for trend $=0.03)$ in the final multivariable analysis.

Conclusion: High midlife intake of the isoflavone genistein is associated with late-life cognitive impairment.
\end{abstract}

Key words: dementia; general population cohort; isoflavones; late-life; midlife; mild cognitive impairment; soy foods

Copyright $\odot 2021$ Thomas Svensson et al. This is an open access article distributed under the terms of Creative Commons Attribution License, which permits unrestricted use, distribution, and reproduction in any medium, provided the original author and source are credited.

\section{INTRODUCTION}

The number of people with dementia in the world is expected to more than double by $2050 .{ }^{1}$ One third of dementia cases could be attributable to modifiable risk factors. ${ }^{2}$ It is therefore crucial to identify any factors that could aid in the early intervention and prevention of cognitive decline, including mild cognitive impairment (MCI), as individuals with MCI progress to dementia at higher rates than those who are cognitively healthy. ${ }^{3}$

Soy and isoflavones, phytoestrogens for which the predominant food source in Asia is soybean, have garnered attention for their beneficial health effects. The consumption of isoflavones is associated with a reduced risk of cerebral and myocardial infarction among women, ${ }^{4}$ and fermented soy products may protect against high blood pressure ${ }^{5}$ and cardiovascular disease mortality. ${ }^{6}$ Contrary to the evidence supporting a cardioprotective effect of dietary isoflavone and soy intake, the findings from observational studies indicate that tofu consumption is inversely associated with memory, ${ }^{7}$ cognitive performance, ${ }^{8}$ and cognitive function. ${ }^{9}$ The only soy food with a reportedly positive effect on cognition is tempeh, a fermented soy product which in one study offset the negative effect of tofu. ${ }^{7}$

Observational studies have considered the habitual consumption of soy/isoflavones concomitantly with the assessment of cognitive function, thereby increasing the risk of introducing reverse causation bias. The long-term effects of isoflavone intake on cognitive impairment have, to the best of our knowledge, been investigated in only two studies, one of which reported a protective effect for women only ${ }^{10}$ and another which reported no association. ${ }^{11}$ Additional studies investigating the association between midlife soy and isoflavone intake and late-life cognitive impairment are therefore warranted.

The purpose of the present study was to investigate, in a Japanese general population cohort, the associations of energyadjusted midlife dietary intake of the isoflavone genistein and soy with cognitive impairment, defined as a composite of late-life MCI and dementia. In accordance with available research, we hypothesize that intake of fermented soy products, as well as high genistein intake, may be inversely associated with cognitive impairment.

Address for correspondence. Norie Sawada, MD, PhD, Epidemiology and Prevention Group, Center for Public Health Sciences, National Cancer Center, 5-1-1 Tsukiji, Chuo-ku, Tokyo 104-0045, Japan (e-mail: nsawada@ncc.go.jp).

JPHC Study group members are listed in Appendix. 


\section{METHODS}

\section{Study population}

The Japan Public Health Center-based prospective (JPHC) Study is a large population-based cohort which was started in 1990 and was conducted in two cohorts; cohort I was initiated in 1990 and cohort II in 1993. The study design has been described in detail elsewhere. ${ }^{12}$ JPHC Study participants were identified using population registries maintained by the local municipalities in 11 public health center (PHC) areas. There were 140,420 individuals identified in the JPHC study at baseline. The study population of the present study were the $12,219 \mathrm{JPHC}$ study participants who in 1990 were between the ages of 40 and 59 and residents of Saku PHC area in Nagano Prefecture (Figure 1). In 2014-2015, 8,827 individuals were considered eligible for an invitation to participate in a mental health screening, following the exclusion of those who had moved out of Saku PHC area, had died, or had not responded to the JPHC Study baseline questionnaire. A total of 1,299 individuals completed the screening.

Following the exclusion of those with an average energyadjusted intake (in kilocalories [kcal]) in the top $(>4,279.4$ $\mathrm{kcal}$ [men] and $>4,418.6 \mathrm{kcal}$ [women]) or bottom $(<1,284.33$ $\mathrm{kcal}$ [men] and $<1,033.1 \mathrm{kcal}$ [women]) percentile $(n=120)$, body mass index (BMI) values $<14 \mathrm{~kg} / \mathrm{m}^{2}$ (indicative of severe underweight and possibility of concomitant disease) or $>60$ $\mathrm{kg} / \mathrm{m}^{2}$ (indicative of a high possibility of unreasonable values) $(n=4)$, missing data for any of the adjusting variables $(n=47)$, or those who had been diagnosed with depression at the mental health screening $(n=92)$, there were 1,036 participants eligible for analyses in the present study. Of the 1,036 eligible participants, 46 individuals were diagnosed with dementia and 346 individuals with MCI at the time of screening.
JPHC study participants were followed-up on three occasions with detailed questionnaires on diet, socioeconomic, and lifestyle factors. The present study used information from all three surveys: baseline (1990-1992), 5-year follow-up (1995), and 10year follow-up (2000).

All participants provided written informed consent to take part in the mental health screening in 2014-2015, and the study has been approved by the Institutional Review Board of the National Cancer Center (approval number: 2013-096).

\section{Soy and isoflavone intake}

Dietary intake of soy and genistein was assessed through the same self-administered 147-item food frequency questionnaire (FFQ) at both the 5-year and 10-year follow-up surveys. For each food item, the pre-specified food consumption frequencies and portion sizes were multiplied to calculate daily food consumption of the respective food item. The daily dietary intake of nutrients was calculated based on the $5^{\text {th }}$ Revised and Enlarged edition of the Standard Tables of Food Composition in Japan. ${ }^{13}$

Eight soy food items were included in the FFQ: miso soup, soymilk, tofu for miso soup, tofu for other dishes, yushidofu (predrained tofu), koyadofu (freeze-dried tofu), aburaage (deep-fried tofu), and natto (fermented soybeans). Detailed consumption frequencies and amount depended on the specific soy food item: miso soup (almost never, 1-3 days/month, 1-2 days/week, 3-4 days/week, 5-6 days/week, or daily, and the number of $150 \mathrm{~mL}$ bowls per day: $<1,1,2,3,4,5,6,7-9$ or $>9$ ), soymilk (almost never, 1-3 times/month, 1-2 times/week, 3-4 times/week, 5-6 times/week, and the number of $200 \mathrm{~mL}$ glasses per day: 1, 2-3, 4-6, 7-9 or >9), and remaining soy food items (frequencies: almost never, 1-3 times/month, 1-2 times/week, 3-4 times/ week, 5-6 times/week, 1 time/day, 2-3 times/day, 4-6 times/

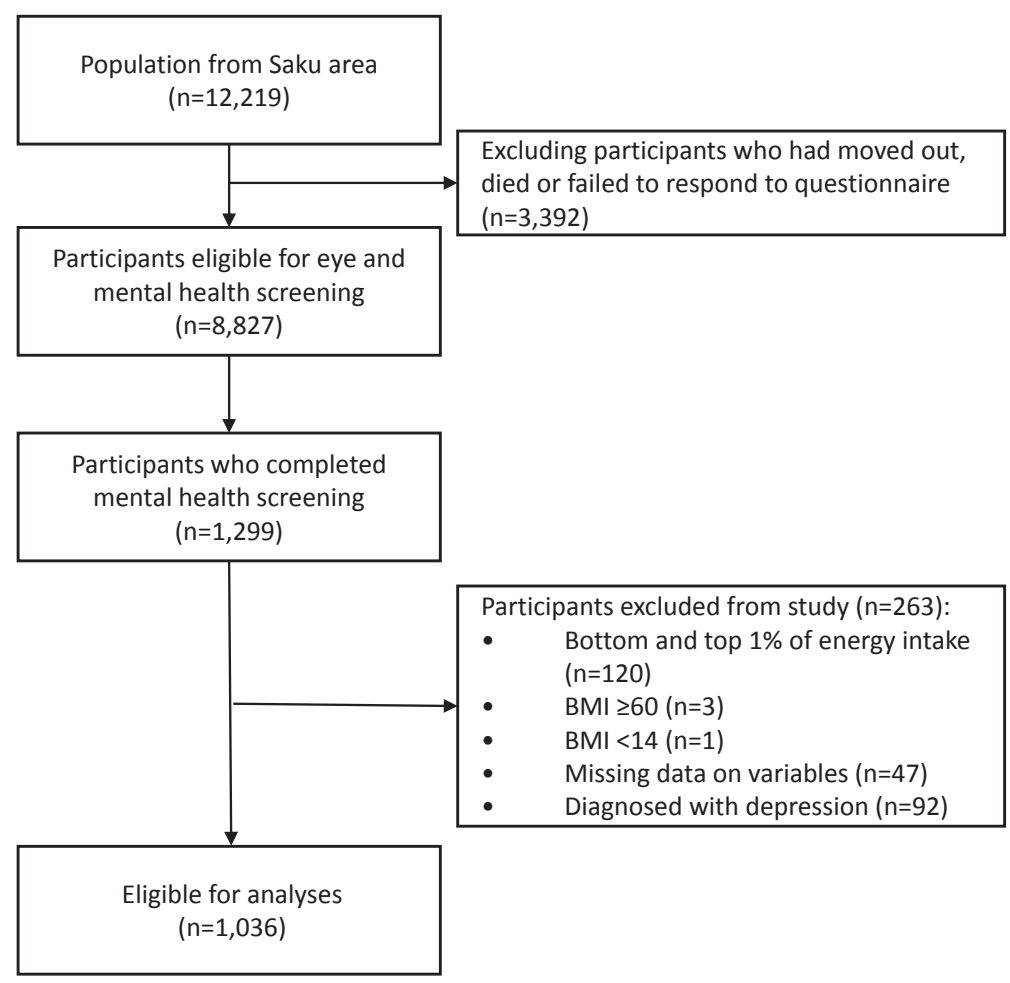

Figure 1. Flowchart for the inclusion and exclusion of study participants 
day and $\geq 7$ times/day, and portion sizes: small [50\% smaller than standard], medium [same as standard], and large [50\% larger than standard]).

The daily intake of genistein and daidzein were based on a specially developed food composition table for isoflavones in Japanese foods. ${ }^{14,15}$ Due to the high correlation between genistein and daidzein, genistein was chosen as representative of isoflavone intake in the present study. The FFQ-derived energy-adjusted genistein intake has been validated in a subsample with consecutive 14- or 28-day dietary records in which Spearman's correlation coefficients between the FFQ and the dietary records were 0.65 for men and 0.55 for women. ${ }^{16}$ Spearman's correlation coefficients for the reproducibility of energy-adjusted genistein intake between the two questionnaires assessed 1 year apart was 0.75 for men and 0.69 for women. ${ }^{16}$

For the purpose of the present study, the sex-specific average energy-adjusted intake of soy foods was calculated based on the 5-year and 10-year FFQs. Food and nutrient intake was logtransformed and adjusted for total energy intake using the residual method. ${ }^{17}$

There were six main exposure groups: overall genistein intake, overall soy food, tofu, miso, natto, and fermented soy (miso and natto combined). For each of the groups, quartiles of energyadjusted intake were constructed based on the cut-off points for participants who were free from MCI and dementia at the time of the mental health screening.

\section{Mild cognitive impairment and dementia}

Cognitive function was assessed by experienced neuropsychologists using the Mini-Mental State Examination (MMSE), ${ }^{18}$ the Wechsler Memory Scale Revised (WMS-R) logical memory I/II subtest, ${ }^{19}$ the clock drawing test, ${ }^{20}$ and the Clinical Dementia Rating (CDR) Scale. ${ }^{21}$ Participants' cognitive function was subsequently categorized in accordance with criteria used in the Japanese Alzheimer's Disease Neuroimaging Initiative (J-ADNI) project, ${ }^{22,23}$ where MCI was defined as amnestic MCI as originally presented by Petersen et al. ${ }^{24}$ Memory impairment was assessed based on an education-adjusted score below the cut-off level on the WMS-R Logical Memory II test (education for $0-9$ years was 2 , for $10-15$ years was 4 , and for $>15$ years was 8). The MMSE cut-off point for dementia was set at 23. Depressive symptoms were assessed using the Center for Epidemiologic Studies Depression Scale (CES-D), ${ }^{25}$ and the Patient Health Questionnaire-9 (PHQ-9). ${ }^{26}$ A trained psychiatrist combined the neuropsychological assessment with a clinical interview to determine the final diagnosis in accordance with the Diagnostic and Statistical Manual of Mental Disorders, 4th. Edition Text Revision (DSM-IV-TR) criteria. $^{27}$

\section{Covariates}

Education was from the baseline questionnaire and categorized as junior high school, high school, or college/vocational school/ other; alcohol consumption (binarized as $<150$ or $\geq 150$ g ethanol per week), smoking status (non-smoker, past smoker, or current smoker), body mass index (BMI in $\mathrm{kg} / \mathrm{m}^{2}$ ), and physical activity (defined as metabolic equivalents) were from the 5-year followup questionnaire; age, prescribed medication (defined as having taken any medications for psychiatric or neurological conditions), self-reported history of diabetes mellitus (yes/no), and selfreported history of stroke (yes/no) were obtained at the time of the mental health screening; All food and nutrient intake (ie, folate, fish, meat, vegetables, fruits, and sodium) were calculated as sex-specific average energy-adjusted intake based on the 5year and 10-year FFQs. Intake was log-transformed and adjusted for total energy intake using the residual method. ${ }^{17}$

\section{Statistical analyses}

Differences in baseline characteristics between quartiles of energy-adjusted genistein intake were determined using the Chi-square test for categorical variables and analysis of variance for continuous variables.

Logistic regression models were used to determine odds ratios (ORs) and 95\% confidence intervals (CIs) for the association between each of the genistein and soy food groups and cognitive impairment. Model 1 was adjusted for age (continuous), sex, education, and energy-adjusted folate intake. Model 2 was additionally adjusted for alcohol consumption, smoking status, BMI (continuous), and physical activity. Model 3 was further adjusted for use of prescribed medication, a self-reported history of diabetes mellitus, and a self-reported history of stroke. Model 4 was additionally adjusted for energy-adjusted intake of fish, meat, vegetables, fruits, and sodium. Missing data was accounted for by listwise deletion. Sensitivity analyses were stratified by sex.

Statistical analyses were performed using SAS (SAS software version 9.4; SAS institute Inc., Cary, NC, USA). $P$-values were two-tailed and considered significant if $P<0.05$.

\section{RESULTS}

Baseline characteristics for complete cases according to quartiles of energy-adjusted genistein intake show that those in the top quartile were older, had the highest intake of folate, fish, fruits, and sodium, and consumed the least amount of meat (Table 1). The mean age and energy-adjusted genistein intake of those without cognitive impairment at the time of screening was 72.3 (standard deviation [SD], 5.4) years, and 26.5 (SD, 13.6) mg/day, respectively.

\section{Cognitive impairment}

At the mental health screening, 392 participants were diagnosed with cognitive impairment, defined as MCI or dementia. The mean age and energy-adjusted genistein intake of those diagnosed with cognitive impairment at the time of screening was 74.5 (SD, 5.6) years, and $29.2(\mathrm{SD}, 16.2) \mathrm{mg} /$ day, respectively.

Compared to those with the lowest energy-adjusted intake (quartile 1) of genistein in model 1, individuals with the highest intake (quartile 4) had significantly higher odds of cognitive impairment (OR 1.58; 95\% CI, 1.08-2.31) (Table 2). The association was only slightly attenuated by the addition of covariates and remained significant also in the fully adjusted model (Model 4: OR $1.51 ; 95 \%$ CI, 1.02-2.24). There was a significant linear trend for the association between genistein intake and cognitive impairment $(P=0.03)$.

Compared to the lowest soy food intake (quartile 1), the highest soy food intake (quartile 4) was significantly associated with cognitive impairment in models 1-3 (Model 1: OR 1.48; 95\% CI, 1.02-2.14) and was abrogated only following the addition of energy-adjusted intakes of meat, fish, vegetables, fruits, and sodium (Model 4: OR 1.41; 95\% CI, 0.97-2.06). The intake of tofu, miso soup, natto, and fermented soy foods was not associated with cognitive impairment in any of the models. 
Table 1. Baseline characteristics according to quartiles of isoflavone intake for all eligible participants $(n=1,036)$

\begin{tabular}{|c|c|c|c|c|c|}
\hline \multirow{2}{*}{ Characteristics } & \multicolumn{4}{|c|}{ Quartile intake of isoflavone (Median intake mg/day) } & \multirow{2}{*}{$P$ value } \\
\hline & Quartile 1 (13.7) & Quartile 2 (21.1) & Quartile 3 (28.5) & Quartile 4 (40.3) & \\
\hline Proportion of participants, $\%$ & 23.9 & 25.7 & 22.7 & 27.7 & \\
\hline Age, ${ }^{\text {b }}$ years, mean $(\mathrm{SD})$ & $72.3(5.4)$ & $73.1(5.6)$ & $73.2(5.5)$ & $73.9(5.6)$ & 0.01 \\
\hline Men (\%) & 39.5 & 44.4 & 40.0 & 42.9 & 0.64 \\
\hline Education, \% & & & & & 0.18 \\
\hline Junior high school & 29.0 & 24.4 & 31.5 & 35.5 & \\
\hline High school & 52.4 & 55.6 & 50.6 & 49.1 & \\
\hline College/vocational school, University or Other & 18.6 & 19.9 & 17.9 & 15.3 & \\
\hline Alcohol consumption, $\%$ & & & & & 0.42 \\
\hline$<150$ g ethanol per week & 79.0 & 74.8 & 74.5 & 79.1 & \\
\hline$\geq 150 \mathrm{~g}$ ethanol per week & 21.0 & 25.2 & 25.5 & 20.9 & \\
\hline Smoking status, $\%$ & & & & & 0.22 \\
\hline Non-smoker & 69.0 & 69.9 & 74.0 & 72.5 & \\
\hline Past smoker & 11.7 & 9.0 & 8.5 & 11.5 & \\
\hline Current smoker $<20$ cigarettes/day & 6.9 & 6.8 & 4.3 & 8.4 & \\
\hline Current smoker $\geq 20$ cigarettes/day & 12.5 & 14.3 & 13.2 & 7.7 & \\
\hline Body Mass Index, kg/m², mean (SD) & $23.2(3.1)$ & $23.4(2.6)$ & $23.4(2.6)$ & $23.6(2.7)$ & 0.54 \\
\hline Physical activity, MET-h/day, mean (SD) & $37.5(9.6)$ & $38.2(10.0)$ & $37.3(9.5)$ & $37.7(10.0)$ & 0.75 \\
\hline History of diabetes mellitus, \% & 8.1 & 8.3 & 10.2 & 10.1 & 0.75 \\
\hline History of stroke, \% & 5.2 & 4.1 & 4.3 & 3.5 & 0.80 \\
\hline Prescribed medication, $\%$ & 10.5 & 9.0 & 8.1 & 12.5 & 0.35 \\
\hline \multicolumn{6}{|l|}{ Energy-adjusted mean dietary intake } \\
\hline Folate, $\mu \mathrm{g} /$ day, mean (SD) & $448.0(135.9)$ & $477.4(129.6)$ & $530.3(126.8)$ & $555.2(125.6)$ & $<0.001$ \\
\hline Meat, g/day, mean (SD) & $72.2(39.7)$ & $71.9(34.6)$ & $72.9(40.7)$ & $63.3(31.1)$ & 0.002 \\
\hline Fish, g/day, mean (SD) & $97.6(47.0)$ & $105.2(50.3)$ & $103.7(41.2)$ & $108.1(44.7)$ & 0.07 \\
\hline Vegetables, g/day, mean (SD) & $287.8(160.2)$ & $321.4(161.1)$ & $355.8(144.3)$ & $347.3(151.1)$ & $<0.001$ \\
\hline Fruits, g/day, mean (SD) & $256.6(167.7)$ & $255.0(157.3)$ & $268.5(154.2)$ & $289.2(150.0)$ & 0.04 \\
\hline Sodium, g/day, mean (SD) & $5.2(1.4)$ & $5.8(1.3)$ & $6.1(1.4)$ & $6.2(1.4)$ & $<0.001$ \\
\hline
\end{tabular}

SD, standard deviation.

${ }^{\mathrm{a}}$ Analysis of Variance for continuous variables; Chi-square test for categorical variables.

${ }^{\mathrm{b}}$ Age at the time of mental health screening.

Table 2. Odds ratios and their confidence intervals of cognitive impairment according to energy-adjusted intake of isoflavone, soy food, tofu, miso soup, and fermented soy products

\begin{tabular}{|c|c|c|c|c|c|}
\hline & \multicolumn{4}{|l|}{ Quartile intake } & \multirow[b]{2}{*}{$P$ for trend } \\
\hline & $\begin{array}{l}\text { Quartile } 1 \\
\text { (Low) }\end{array}$ & Quartile 2 & Quartile 3 & $\begin{array}{l}\text { Quartile } 4 \\
\text { (High) }\end{array}$ & \\
\hline \multicolumn{6}{|l|}{ Isoflavone } \\
\hline Number (Events) & $248(84)$ & $266(95)$ & $235(86)$ & $287(127)$ & \\
\hline $\mathrm{mg} /$ day, median [range] & $13.7[4.2-17.2]$ & $21.1[17.2-24.8]$ & $28.5[24.7-32.6]$ & $40.3[32.6-161.3]$ & \\
\hline $\begin{array}{l}\text { Model } 1^{\mathrm{a}} \\
\text { OR }(95 \% \mathrm{CI})\end{array}$ & Reference & $1.06(0.73-1.54)$ & $1.17(0.79-1.74)$ & $1.58 *(1.08-2.31)$ & 0.01 \\
\hline $\begin{array}{l}\text { Model } 2^{\mathrm{b}} \\
\text { OR }(95 \% \mathrm{CI})\end{array}$ & Reference & $1.03(0.70-1.50)$ & $1.18(0.79-1.76)$ & $1.57^{*}(1.07-2.31)$ & 0.01 \\
\hline $\begin{array}{l}\text { Model 3 } \\
\text { OR }(95 \% \text { CI })\end{array}$ & Reference & $1.04(0.71-1.52)$ & $1.19(0.80-1.78)$ & $1.56^{*}(1.06-2.30)$ & 0.02 \\
\hline $\begin{array}{l}\text { Model } 4^{\mathrm{d}} \\
\text { OR }(95 \% \text { CI })\end{array}$ & Reference & $1.01(0.69-1.48)$ & $1.15(0.77-1.72)$ & $1.51^{*}(1.02-2.24)$ & 0.03 \\
\hline \multicolumn{6}{|l|}{ Soy food } \\
\hline Number (Events) & $260(97)$ & $225(67)$ & $250(88)$ & $301(140)$ & \\
\hline $\mathrm{g} /$ day, median [range] & 38.6 [7.9-50.9] & $58.5[47.8-70.1]$ & 78.4 [65.5-92.9] & 115.8 [87.0-966.4] & \\
\hline $\begin{array}{l}\text { Model } 1^{\mathrm{a}} \\
\text { OR }(95 \% \mathrm{CI})\end{array}$ & Reference & $0.72(0.49-1.07)$ & $0.89(0.61-1.31)$ & $1.48^{*}(1.02-2.14)$ & 0.01 \\
\hline $\begin{array}{l}\text { Model } 2^{\mathrm{b}} \\
\text { OR }(95 \% \text { CI })\end{array}$ & Reference & $0.72(0.48-1.06)$ & $0.88(0.60-1.30)$ & $1.48^{*}(1.02-2.14)$ & 0.02 \\
\hline $\begin{array}{l}\text { Model 3c } \\
\text { OR }(95 \% \text { CI) }\end{array}$ & Reference & $0.71(0.48-1.06)$ & $0.87(0.59-1.28)$ & $1.46^{*}(1.01-2.13)$ & 0.02 \\
\hline $\begin{array}{l}\text { Model } 4^{\mathrm{d}} \\
\text { OR }(95 \% \mathrm{CI})\end{array}$ & Reference & $0.68(0.46-1.02)$ & $0.82(0.55-1.21)$ & $1.41(0.97-2.06)$ & 0.03 \\
\hline
\end{tabular}




\begin{tabular}{|c|c|c|c|c|c|}
\hline & \multicolumn{4}{|l|}{ Quartile intake } & \multirow[b]{2}{*}{$P$ for trend } \\
\hline & $\begin{array}{l}\text { Quartile } 1 \\
\text { (Low) }\end{array}$ & Quartile 2 & Quartile 3 & $\begin{array}{l}\text { Quartile } 4 \\
\text { (High) }\end{array}$ & \\
\hline \multicolumn{6}{|l|}{ Tofu } \\
\hline Number (Events) & $248(86)$ & $247(82)$ & $287(124)$ & $254(100)$ & \\
\hline g/day, median [range] & $11.3[-0.27-16.8]$ & $21.6[15.9-27.2]$ & $32.4[26.1-41.7]$ & $55.2[40.0-186.9]$ & \\
\hline $\begin{array}{l}\text { Model } 1^{\mathrm{a}} \\
\text { OR }(95 \% \mathrm{CI})\end{array}$ & Reference & $0.95(0.65-1.39)$ & $1.41(0.98-2.02)$ & $1.16(0.79-1.68)$ & 0.16 \\
\hline $\begin{array}{l}\text { Model } 2^{\mathrm{b}} \\
\text { OR }(95 \% \mathrm{CI})\end{array}$ & Reference & $0.96(0.66-1.42)$ & $1.40(0.97-2.01)$ & $1.15(0.79-1.68)$ & 0.18 \\
\hline $\begin{array}{l}\text { Model 3c } \\
\text { OR }(95 \% \text { CI) }\end{array}$ & Reference & $0.95(0.65-1.41)$ & $1.39(0.96-2.01)$ & $1.15(0.79-1.68)$ & 0.18 \\
\hline $\begin{array}{l}\text { Model } 4^{\mathrm{d}} \\
\text { OR }(95 \% \mathrm{CI})\end{array}$ & Reference & $0.95(0.64-1.40)$ & $1.33(0.92-1.93)$ & $1.13(0.77-1.67)$ & 0.24 \\
\hline \multicolumn{6}{|l|}{ Miso soup } \\
\hline Number (Events) & $261(100)$ & $245(82)$ & $250(90)$ & $280(120)$ & \\
\hline $\mathrm{mL} /$ day, median [range] & $36.1[-0.12-76.2]$ & $87.8[58.7-135.8]$ & 141.4 [105.8-187.9] & 202.8 [144.8-639.2] & \\
\hline $\begin{array}{l}\text { Model 1 } 1^{\mathrm{a}} \\
\text { OR }(95 \% \mathrm{CI})\end{array}$ & Reference & $0.79(0.54-1.14)$ & $0.83(0.57-1.20)$ & $1.17(0.82-1.67)$ & 0.33 \\
\hline $\begin{array}{l}\text { Model } 2^{\mathrm{b}} \\
\text { OR }(95 \% \mathrm{CI})\end{array}$ & Reference & $0.78(0.54-1.14)$ & $0.81(0.56-1.18)$ & $1.15(0.80-1.65)$ & 0.40 \\
\hline $\begin{array}{l}\text { Model 3c } \\
\text { OR }(95 \% \text { CI) }\end{array}$ & Reference & $0.79(0.54-1.15)$ & $0.82(0.56-1.19)$ & $1.19(0.83-1.71)$ & 0.31 \\
\hline $\begin{array}{l}\text { Model } 4^{\mathrm{d}} \\
\text { OR }(95 \% \mathrm{CI})\end{array}$ & Reference & $0.78(0.53-1.15)$ & $0.80(0.54-1.19)$ & $1.19(0.81-1.75)$ & 0.38 \\
\hline \multicolumn{6}{|l|}{ Natto } \\
\hline Number (Events) & $257(97)$ & $254(91)$ & 247 (89) & $278(115)$ & \\
\hline $\mathrm{g} /$ day, median [range] & $3.5[-0.46-6.3]$ & $8.1[5.6-10.7]$ & $13.2[9.5-18.3]$ & $23.9[16.2-83.1]$ & \\
\hline $\begin{array}{l}\text { Model } 1^{\mathrm{a}} \\
\text { OR }(95 \% \mathrm{CI})\end{array}$ & Reference & $0.94(0.65-1.36)$ & $0.93(0.64-1.35)$ & $1.18(0.81-1.70)$ & 0.39 \\
\hline $\begin{array}{l}\text { Model } 2^{\mathrm{b}} \\
\text { OR }(95 \% \mathrm{CI})\end{array}$ & Reference & $0.94(0.65-1.37)$ & $0.93(0.64-1.36)$ & $1.20(0.83-1.74)$ & 0.34 \\
\hline $\begin{array}{l}\text { Model 3c } \\
\text { OR (95\% CI) }\end{array}$ & Reference & $0.92(0.63-1.34)$ & $0.93(0.63-1.36)$ & $1.20(0.83-1.75)$ & 0.32 \\
\hline $\begin{array}{l}\text { Model } 4^{\mathrm{d}} \\
\text { OR }(95 \% \mathrm{CI})\end{array}$ & Reference & $0.91(0.62-1.34)$ & $0.90(0.61-1.32)$ & $1.18(0.81-1.72)$ & 0.41 \\
\hline \multicolumn{6}{|l|}{$\begin{array}{l}\text { Fermented soy (Natto } \\
\text { +Miso) }\end{array}$} \\
\hline Number (Events) & $245(83)$ & $255(95)$ & $260(96)$ & $276(118)$ & \\
\hline $\mathrm{g} /$ day, median [range] & $46.7[3.6-86.0]$ & 97.4 [70.3-146.7] & $155.5[120.0-199.3]$ & 216.0 [163.2-647.5] & \\
\hline $\begin{array}{l}\text { Model } 1^{\mathrm{a}} \\
\text { OR }(95 \% \mathrm{CI})\end{array}$ & Reference & $1.14(0.78-1.66)$ & $1.06(0.73-1.54)$ & $1.41(0.97-2.03)$ & 0.10 \\
\hline $\begin{array}{l}\text { Model 2b } \\
\text { OR }(95 \% \text { CI) }\end{array}$ & Reference & $1.11(0.76-1.62)$ & $1.04(0.71-1.51)$ & $1.37(0.94-1.98)$ & 0.14 \\
\hline $\begin{array}{l}\text { Model 3c } \\
\text { OR }(95 \% \mathrm{CI})\end{array}$ & Reference & $1.11(0.76-1.63)$ & $1.04(0.71-1.52)$ & $1.41(0.97-2.05)$ & 0.10 \\
\hline $\begin{array}{l}\text { Model } 4^{\mathrm{d}} \\
\text { OR }(95 \% \mathrm{CI})\end{array}$ & Reference & $1.09(0.74-1.61)$ & $1.02(0.69-1.52)$ & $1.38(0.93-2.07)$ & 0.16 \\
\hline
\end{tabular}

CI, confidence interval; OR, odds ratio.

${ }^{*} P<0.05$;

${ }^{\mathrm{a}}$ Model 1 is adjusted for age, gender, education and energy-adjusted folate intake;

${ }^{\mathrm{b}}$ Model 2 is additionally adjusted for alcohol consumption, smoking, body mass index, and physical activity;

${ }^{\mathrm{c}}$ Model 3 is additionally adjusted for history of diabetes mellitus, history of stroke, and use of prescribed medications;

${ }^{\mathrm{d}}$ Model 4 is additionally adjusted for energy-adjusted intake of fish, meat, vegetables, fruits, and sodium.

\section{Sex-stratified analyses}

Of the 433 men, 189 were diagnosed with cognitive impairment at the mental health screening. The mean age and energyadjusted genistein intake of men diagnosed with cognitive impairment was 74.7 (SD, 5.6) years, and 28.6 (SD, 13.1) $\mathrm{mg} /$ day, respectively.
Compared to men with the lowest energy-adjusted intake (quartile 1) of genistein in model 1, men with the highest intake (quartile 4) had significantly higher odds of cognitive impairment (OR 1.93; 95\% CI, 1.06-3.52) (eTable 1). The association was abrogated in models 2-4 with non-significant associations throughout (Model 4: OR 1.68; 95\% CI, 0.88-3.22). Compared 
to the lowest intake of fermented soy (quartile 1), the highest intake (quartile 4) of fermented soy was significantly associated with cognitive impairment in men in model 1 (OR 1.84; 95\% CI, 1.03-3.29) and model 3 (OR 1.89; 95\% CI, 1.02-3.47) but was ultimately non-significant in the fully adjusted model (Model 4: OR 1.94; 95\% CI, 0.98-3.85). The intake of soy foods in general, tofu, miso, and natto was not associated with cognitive impairment in men.

Of the 603 women, 203 were diagnosed with cognitive impairment at the mental health screening. The mean age and energy-adjusted genistein intake of women diagnosed with cognitive impairment was 74.4 (SD, 5.6) years and 29.8 (SD, 18.6) $\mathrm{mg} /$ day, respectively. Compared to the lowest miso intake (quartile 1), those with high miso intake (quartile 3) had significantly lower odds of cognitive impairment (Model 1: OR 0.56; 95\% CI, 0.33-0.95) (eTable 2). The association was only slightly attenuated by the addition of covariates and remained significant also in the fully adjusted model (Model 4: OR 0.54; 95\% CI, 0.31-0.93). The intake of genistein, soy foods in general, tofu, natto, or fermented soy was not associated with cognitive impairment in women.

\section{DISCUSSION}

This study has shown that individuals with the highest midlife intake (quartile 4) of the isoflavone genistein have an approximately $50 \%$ higher odds of late-life cognitive impairment compared to those with the lowest midlife intake (quartile 1).

Isoflavone and soy intake in Japan is among the highest in Asia, ${ }^{28}$ and our study has allowed us to study the association of long-term and high-dose midlife genistein and soy intake on late-life cognitive impairment. The major limitation of most observational studies investigating the association of soy and isoflavones on cognitive function is their concomitant assessment of both exposure and outcome. Individuals who suffer from cognitive impairment may not accurately recall their food intake or may have changed dietary habits as a direct consequence of their deteriorating health. This would be particularly important when assessing late-life participants, who may have a high prevalence of comorbidities. To the best of our knowledge, there is only one other observational study that has investigated midlife soy food intake, specifically tofu, on late-life cognitive function. ${ }^{9}$ Overall, our results are supported by previous studies, which suggest a detrimental effect of dietary soy intake on cognition..$^{7-9}$ For overall soy intake, it was only in our final multivariable model where the highest soy intake became non-significantly associated with cognitive impairment. This abrogation suggests that the association between soy intake and cognitive impairment must be considered in the context of important dietary confounders; high vegetable and fruit intake may offset any risk of cognitive impairment, whereas sodium intake would be an important confounder with regards to cardiovascular risk.

In this study, we found no association between tofu intake and cognitive impairment. Hogervorst et $\mathrm{al}^{7}$ suggested that the detrimental effects of tofu on memory performance could be due to the use of formaldehyde as a preservative. If this indeed is the true cause of the association between tofu and cognitive function, even high tofu intake over many years would not constitute a health risk in Japan given that the use of formaldehyde as a preservative is prohibited. Additionally, in the study by White et al, the effect of midlife tofu intake explained only a fraction
(2.3\%) of the variance compared to the combined effects of age, education, and a history of stroke $(27.8 \%) .{ }^{9}$ Consequently, the explanatory effect of tofu on cognitive impairment could be so small that it is already accounted for in our well-adjusted study.

One study has reported an inverse association between fermented soy products and cognitive impairment due to the higher concentration of folate, ${ }^{29}$ which is associated with a reduced risk of dementia. ${ }^{30}$ In contrast, in our main analysis, we found no association between midlife intake of fermented soy foods and late-life cognitive impairment. There are, however, two noteworthy results in our sex-stratified analyses: first, among men, intake of fermented soy in the highest quartile was significantly and positively associated with cognitive impairment in two of the models (Model 1 and Model 3). This suggests that the introduction of history of disease, in particular of stroke, in Model 3 results in negative confounding. Stroke is associated with a decline in cognitive function ${ }^{31}$ and increased risk of dementia. ${ }^{32}$ These significant associations were, however, abrogated when adjusting for important dietary confounders in the final model. Second, among women, there was an inverse association between the second highest quartile of midlife miso intake and late-life cognitive impairment. This finding is somewhat difficult to explain without speculation, in particular as the highest intake (quartile 4) of miso shows no association with the outcome. Moreover, the inverse association occurs only for one of the fermented soy products and not for the other, natto, or for the total intake of fermented soy. Taken together, this tells us that it is not a higher amount of fermented soy that is beneficial, which in turn is contrary to the effects expected with increased folate intake. Instead, one mechanism that could possibly help explain the results among women considers the estrogen-like properties of the isoflavones genistein and daidzein and their classification as phytoestrogens. ${ }^{33}$ Although estrogens may be neuroprotective and have beneficial effects on both learning and memory, ${ }^{34}$ high concentrations of phytoestrogens may have anti-estrogenic effects as they bind to the estrogen receptors and thus compete with endogenous estrogen. ${ }^{35}$ Moreover, genistein and daidzein are predominantly found in fermented soy foods ${ }^{36}$ and daidzein has a biologically active metabolite, equol, which is produced by human intestinal bacteria. ${ }^{37}$ This could result in an increased bioavailability of isoflavones and consequently phytoestrogens in individuals who consume high amounts of fermented soy foods, as opposed to those who consume mostly non-fermented soy foods.

There are, to the best of our knowledge, only two studies that have investigated the long-term associations of isoflavone intake on cognitive impairment. These studies report either a protective effect only for women ${ }^{10}$ or no association, ${ }^{11}$ which are contrary to our own results. Our main findings instead show that high midlife intake of the isoflavone genistein is associated with cognitive impairment. Nevertheless, any recommendation to reduce the consumption of soy foods and isoflavones must be considered in the context of other studies, as well as the beneficial effects of isoflavones on cardiovascular health. ${ }^{4-6}$ In the present study, it was only the very highest level of genistein intake which was significantly associated with higher odds of cognitive impairment, indicating that only a very high consumption would have a detrimental effect. The overall high consumption of soy foods in Japan means that individuals who are in the top quartile of genistein consumption in this study would almost certainly be outliers in most other countries. Second, the effect of isoflavones 
must not necessarily be correlated with their consumed amount, as the inter-individual bioavailability of isoflavones may differ. In some individuals, daidzein is converted to equol by intestinal bacterial metabolism. ${ }^{38}$ It is suggested that those who are "equol producing" may constitute a sub-population with regards to the efficacy of soy foods on health outcomes. ${ }^{38}$ Indeed, one small cross-sectional Japanese study found that being an equol producer was a risk factor for MCI, ${ }^{39}$ which may support our findings. Future studies investigating the association between isoflavones and cognitive impairment should include and stratify participants on their equol-producing status. Third, the positive association between genistein and cognitive impairment was not seen in sexstratified analyses. The direction of the associations in both men and women pointed towards increased odds of cognitive impairment with the highest genistein intake. However, the analyses also revealed that, among women, a "moderate" (quartile 2) or "high" intake (quartile 3) pointed towards an inverse association, albeit without any statistical significance. This suggests that larger study samples are required to reach sufficient statistical power and that the associations may be different between men and women. Future studies with sufficient sample sizes are required to investigate this further.

This study has a few limitations: we cannot exclude selection bias given that only approximately $15 \%$ of invited participants completed the mental health screening. We cannot with certainty state that participants were cognitively healthy at baseline; however, the likelihood of a cognitively impaired person completing the entire follow-up period with all follow-up surveys is very small. Generalization of results to other populations may be limited, as the dietary intake of soy food and genistein in Japan is substantially higher than in western countries. Moreover, although we have adjusted our analyses for age, we cannot exclude an age effect where individuals who consume a high amount of soy and isoflavone may live to a higher age where they develop cognitive impairment. We were also unable to test for equol-producing status, an important factor for the bioavailability of isoflavones, which may further limit generalizability. Although sex-stratified analyses were conducted, the results indicate that the strata may be too small to draw any wider conclusions based on the results. Future studies are encouraged to conduct sex-stratified analyses to further investigate the effect of midlife intake of phytoestrogens on cognitive impairment in women. Finally, due to the observational nature of the study, causality cannot be determined.

This study also has a number of considerable strengths: first, it is a study where genistein and soy intake was assessed at midlife, thereby minimizing any reverse causation bias. Second, the detailed questionnaire of the JPHC Study allows us to adjust for a large number of known confounders. Third, the soy and genistein intake was energy-adjusted and averaged over two assessments 5 years apart. This provides a stable and continuous exposure compared to using just a single time point. Finally, cognitive impairment was classified by a trained psychiatrist in accordance with DSM-IV-TR criteria.

\section{Conclusion}

High midlife intake of the isoflavone genistein is associated with late-life cognitive impairment.

\section{ACKNOWLEDGEMENTS}

Thomas Svensson had full access to all the data in the study and takes responsibility for the integrity of the data and the accuracy of the data analysis.

Ethics approval and consent to participate: All participants provided written informed consent to take part in the mental health screening in 2014-2015, and the study has been approved by the Institutional Review Board of the National Cancer Center (approval number: 2013-096).

Financial support: This study was supported by the National Cancer Center Research and Development Fund (since 2011) and a Grant-in-Aid for Cancer Research from the Ministry of Health, Labour and Welfare of Japan (from 1989 to 2010) and Grantin-Aid from the Ministry of Agriculture, Forestry and Fisheries (MAFF-CPS-2016-1-1).

Author contribution statement: TS, NS, MM, and ST were responsible for the conception and design of the study. TS analyzed and interpreted the data. NS, MM, SN, RS, ST assisted with the interpretation of the data. TS drafted the manuscript. NS, MM, SN, RS, ST critically revised the manuscript for important intellectual content. All authors approved the final version of the manuscript.

Conflicts of interest: TS, NS, SN, RS, and ST declare no conflicts of interest. MM declares having received grants from Daiichi Sankyo, Eisai, Pfizer, Shionogi, Takeda, Tanabe Mitsubishi and Tsumura, and payment or honoraria from Byer Pharmaceutical, Daiichi Sankyo, Dainippon-Sumitomo Pharma, Eisai, Eli Lilly, Fuji Film RI Pharma, Hisamitsu Pharmaceutical, Janssen Pharmaceutical, Kyowa Pharmaceutical, Mochida Pharmaceutical, MSD, Mylan EPD, Nihon Medi-physics, Nippon Chemipher, Novartis Pharma, Ono Yakuhin, Otsuka Pharmaceutical, Pfizer, Santen Pharmaceutical, Shire Japan, Takeda Yakuhin, Tsumura, and Yoshitomi Yakuhin.

\section{APPENDIX A. SUPPLEMENTARY DATA}

Supplementary data related to this article can be found at https:// doi.org/10.2188/jea.JE20210199.

\section{REFERENCES}

1. Prince M, Wimo A, Guerchet M, Ali GC, Wu Y, AM. P. World Alzheimer Report 2015: The global impact of dementia. An analysis of prevalence, incidence, costs and trends. London: Alzheimer's Disease International; 2015.

2. de Bruijn RF, Bos MJ, Portegies ML, et al. The potential for prevention of dementia across two decades: the prospective, population-based Rotterdam Study. BMC Med. 2015;13:132.

3. Ganguli M, Snitz BE, Saxton JA, et al. Outcomes of mild cognitive impairment by definition: a population study. Arch Neurol. 2011; 68:761-767.

4. Kokubo Y, Iso H, Ishihara J, Okada K, Inoue M, Tsugane S; JPHC Study Group. Association of dietary intake of soy, beans, and isoflavones with risk of cerebral and myocardial infarctions in Japanese populations: the Japan Public Health Center-based (JPHC) study cohort I. Circulation. 2007;116:2553-2562.

5. Nozue M, Shimazu T, Sasazuki S, et al. Fermented soy product intake is inversely associated with the development of high blood pressure: the Japan Public Health Center-Based Prospective Study. J Nutr. 2017;147:1749-1756.

6. Nagata C, Wada K, Tamura T, et al. Dietary soy and natto intake and cardiovascular disease mortality in Japanese adults: the Takayama study. Am J Clin Nutr. 2017;105:426-431.

7. Hogervorst E, Sadjimim T, Yesufu A, Kreager P, Rahardjo TB. High tofu intake is associated with worse memory in elderly Indonesian men and women. Dement Geriatr Cogn Disord. 2008; 
26:50-57.

8. Xu X, Xiao S, Rahardjo TB, Hogervorst E. Tofu intake is associated with poor cognitive performance among community-dwelling elderly in China. J Alzheimers Dis. 2015;43:669-675.

9. White LR, Petrovitch H, Ross GW, et al. Brain aging and midlife tofu consumption. J Am Coll Nutr. 2000;19:242-255.

10. Nakamoto M, Otsuka R, Nishita Y, et al. Soy food and isoflavone intake reduces the risk of cognitive impairment in elderly Japanese women. Eur J Clin Nutr. 2018;72:1458-1462.

11. Talaei M, Feng L, Yuan JM, Pan A, Koh WP. Dairy, soy, and calcium consumption and risk of cognitive impairment: the Singapore Chinese Health Study. Eur J Nutr. 2020;59:1541-1552.

12. Tsugane S, Sawada N. The JPHC study: design and some findings on the typical Japanese diet. Jpn J Clin Oncol. 2014;44:777-782.

13. Ministry of Education, Culture, Sports, Science and Technology. Standard Tables of Food Composition in Japan, 5th revised and enlarged edition. Tokyo: National Printing Bureau; 2005.

14. Kimira M, Arai Y, Shimoi K, Watanabe S. Japanese intake of flavonoids and isoflavonoids from foods. J Epidemiol. 1998;8:168175.

15. Arai Y, Watanabe S, Kimira M, Shimoi K, Mochizuki R, Kinae N. Dietary intakes of flavonols, flavones and isoflavones by Japanese women and the inverse correlation between quercetin intake and plasma LDL cholesterol concentration. J Nutr. 2000;130:2243-2250.

16. Akhter M, Inoue M, Kurahashi N, Iwasaki M, Sasazuki S, Tsugane S; Japan Public Health Center-Based Prospective Study Group. Dietary soy and isoflavone intake and risk of colorectal cancer in the Japan public health center-based prospective study. Cancer Epidemiol Biomarkers Prev. 2008;17:2128-2135.

17. Willett W, Stampfer MJ. Total energy intake: implications for epidemiologic analyses. Am J Epidemiol. 1986;124:17-27.

18. Folstein MF, Folstein SE, McHugh PR. "Mini-mental state". A practical method for grading the cognitive state of patients for the clinician. J Psychiatr Res. 1975;12:189-198.

19. Elwood RW. The Wechsler Memory Scale-Revised: psychometric characteristics and clinical application. Neuropsychol Rev. 1991;2: 179-201.

20. Agrell B, Dehlin O. The clock-drawing test. Age Ageing. 1998;27: 399-403.

21. Hughes CP, Berg L, Danziger WL, Coben LA, Martin RL. A new clinical scale for the staging of dementia. Br J Psychiatry. 1982;140: 566-572.

22. Fujishima M, Kawaguchi A, Maikusa N, Kuwano R, Iwatsubo T, Matsuda H; Japanese Alzheimer's Disease Neuroimaging Initiative (ADNI); Japanese Alzheimer's Disease Neuroimaging Initiative (JADNI). Sample Size Estimation for Alzheimer's Disease Trials from Japanese ADNI Serial Magnetic Resonance Imaging. J Alzheimers
Dis. 2017;56:75-88.

23. Iwatsubo T. Japanese Alzheimer's Disease Neuroimaging Initiative: present status and future. Alzheimers Dement. 2010;6:297-299.

24. Petersen RC, Smith GE, Waring SC, Ivnik RJ, Tangalos EG, Kokmen E. Mild cognitive impairment: clinical characterization and outcome. Arch Neurol. 1999;56:303-308.

25. Radloff LS. The CES-D Scale: a self-report depression scale for research in the general population. Appl Psychol Meas. 1977;1:385401.

26. Kroenke K, Spitzer RL, Williams JB. The PHQ-9: validity of a brief depression severity measure. J Gen Intern Med. 2001;16:606-613.

27. Diagnostic and statistical manual of mental disorders: DSM-IV-TR. Washington, DC: American Psychiatric Association, 2000 4. ed./ text revision; 2000.

28. Messina M, Nagata C, Wu AH. Estimated Asian adult soy protein and isoflavone intakes. Nutr Cancer. 2006;55:1-12.

29. Mo H, Kariluoto S, Piironen V, et al. Effect of soybean processing on content and bioaccessibility of folate, vitamin B12 and isoflavones in tofu and tempe. Food Chem. 2013;141:2418-2425.

30. Ravaglia G, Forti P, Maioli F, et al. Homocysteine and folate as risk factors for dementia and Alzheimer disease. Am J Clin Nutr. 2005; 82:636-643.

31. Levine DA, Galecki AT, Langa KM, et al. Trajectory of cognitive decline after incident stroke. JAMA. 2015;314:41-51.

32. Savva GM, Stephan BC; Alzheimer's Society Vascular Dementia Systematic Review Group. Epidemiological studies of the effect of stroke on incident dementia: a systematic review. Stroke. 2010;41: e41-e46.

33. Mostrom M, Evans TJ. Phytoestrogens. In: Gupta RC, editor Reproductive and Developmental Toxicology. 2011:707-722.

34. Kassam S, Hogervorst E. Effects of Dietary Estrogens on Dementia. Estrogens and Memory. New York: Oxford University Press; 2020:359-377.

35. Rosselli M, Reinhart K, Imthurn B, Keller PJ, Dubey RK. Cellular and biochemical mechanisms by which environmental oestrogens influence reproductive function. Hum Reprod Update. 2000;6:332350

36. Wang $\mathrm{H}$, Murphy PA. Isoflavone content in commercial soybean foods. J Agric Food Chem. 1994;42:1666-1673.

37. Lampe JW. Is equol the key to the efficacy of soy foods? Am J Clin Nutr. 2009;89:1664S-1667S.

38. Setchell KD, Brown NM, Lydeking-Olsen E. The clinical importance of the metabolite equol-a clue to the effectiveness of soy and its isoflavones. J Nutr. 2002;132:3577-3584.

39. Igase M, Igase K, Tabara Y, Ohyagi Y, Kohara K. Cross-sectional study of equol producer status and cognitive impairment in older adults. Geriatr Gerontol Int. 2017;17:2103-2108. 